\title{
A Double Nonmonotone Quasi-Newton Method for Nonlinear Complementarity Problem Based on Piecewise NCP Functions
}

\author{
Zhensheng Yu $\mathbb{D}^{1}{ }^{1}$ Zilun Wang ${ }^{D},{ }^{1}$ and $K e ~ S u^{2}$ \\ ${ }^{1}$ College of Science, University of Shanghai for Science and Technology, Shanghai 200093, China \\ ${ }^{2}$ College of Mathematics and Information Science, Hebei University, Hebei 071002, China \\ Correspondence should be addressed to Zhensheng Yu; zhsh-yu@163.com
}

Received 22 October 2020; Revised 12 November 2020; Accepted 26 November 2020; Published 16 December 2020

Academic Editor: Guoqiang Wang

Copyright (C) 2020 Zhensheng Yu et al. This is an open access article distributed under the Creative Commons Attribution License, which permits unrestricted use, distribution, and reproduction in any medium, provided the original work is properly cited.

In this paper, a double nonmonotone quasi-Newton method is proposed for the nonlinear complementarity problem. By using 31 piecewise and 4-1 piecewise nonlinear complementarity functions, the nonlinear complementarity problem is reformulated into a smooth equation. By a double nonmonotone line search, a smooth Broyden-like algorithm is proposed, where a single solution of a smooth equation at each iteration is required with the reduction in the scale of the calculation. Under suitable conditions, the global convergence of the algorithm is proved, and numerical results with some practical applications are given to show the efficiency of the algorithm.

\section{Introduction}

In this paper, we consider the following nonlinear complementarity problem (NCP): find $x \in R^{n}$ such that

$$
x \geq 0, F(x) \geq 0, x^{T} F(x)=0 .
$$

where $F: R^{n} \longrightarrow R^{n}$ is continuously differentiable and the superscript $T$ denotes the transpose operator. When $F$ is linear, problem (1) reduces to a linear complementarity problem (LCP). Throughout this paper, the solution set of problem (1), denoted by $X^{*}$, is assumed to be nonempty.

Nonlinear complementarity problems arisen in many practical applications, for example, the KKT systems of mathematical programming problem, the economic equilibrium, the engineering design problem, can be reformulated into the NCP [1-3].

During the past decades, various efficient numerical algorithms are proposed to solve the NCP. One of the most effective methods is to transform the NCP into the semismooth equations (based on nonlinear complementarity function, NCP function) so that the semismooth Newtontype method can be deployed. The most well-known NCP functions are the Fischer-Burmeister function [4] (FB NCP function) and the modified FB NCP function [5]. Sun and Qi [6] proposed several NCP functions, investigated their properties, and provided a numerical comparison between the behavior of different NCP functions. Based on NCP functions, some kinds of algorithm are designed, see, for example, [7-11].

Another well-known class of algorithm is the smoothing algorithm. The main idea of smoothing algorithm is to reformulate the NCP to smooth equations by introducing the smoothing NCP functions. Some smoothing NCP functions and the corresponding algorithms can be found in [12-15].

Besides the NCP functions mentioned above, a 3-1 piecewise NCP function was proposed by Liu et al. [16], using it to solve the inequality-constrained nonlinear optimization. The advantage of the 3-1 piecewise lies in the absence of the smoothing parameter. Motivated by the 3-1 piecewise NCP function, Su and Yang $[17,18]$ developed smooth-based Newton algorithms with nonmonotone line search for nonlinear complementarity and generalized nonlinear complementarity problems. Different from the 
previous methods, the authors introduced independent variable quantities to simplify the algorithm, reducing the amount of calculation without using the smoothing parameter.

Smoothing procedure allows one to use successful quasiNewton approaches, and there are many quasi Newton methods available for the nonlinear complementarity problems based on some smoothing functions [19-26].

In this paper, we will construct a 3-1 piecewise and 4-1 piecewise NCP functions and develop a double nonmonotone quasi Newton method to solve the nonlinear complementarity problems. Based on the piecewise NCP functions, the nonlinear complementarity problem is transformed into the smooth equation. Moreover, we only solve one smooth equation at each iteration. In order to get the better numerical results, a double nonmonotone line search is used by combining with the Broyden-like algorithm. Consequently, the omission of the parameter $\mu$ and the single calculation of the Jacobian matrix at each iteration have led to the simplicity and flexibility of this approach. Furthermore, let $t=F(x)$ as an independent variable, which has no relationship with $x$, ensures the realization of our algorithm easier. Our algorithm is proved to be well-defined and globally convergent under suitable conditions. At the end of the paper, we give numerical results to prove the effectiveness of the algorithm. This paper is organized as follows: the piecewise linear NCP functions are introduced in Section 1. The double nonmonotone line search with quasi-Newton method is given in Section 2. In Section 3, the convergence properties of the algorithm are presented. We give some numerical results in Section 4, and the conclusion is drawn in Section 5.

\section{Algorithm Analysis}

To describe our algorithm, we first give the definitions of NCP function and $P_{0}$ function. We assume that $F: R^{n} \longrightarrow R^{n}$ is a continuously differentiable $P_{0}$-function; if, for all $x, y \in R^{n}$ with $x \neq y$, there exists an index $i$ such that

$$
\left(x_{i}-y_{i}\right)^{T}\left[F_{i}(x)-F_{i}(y)\right] \geq 0, \quad x_{i} \neq y_{i},
$$

and we regard a pair $(a, b) \in R^{2}$ as an NCP pair if $a \geq 0, b \geq 0$, and $a^{T} b=0$; a function $\Phi: R^{2} \longrightarrow R$ is called an NCP function, and we have $\Phi(a, b)=0$ if and only if $(a, b)$ is a NCP pair.

In what follows, we first introduce the $3-1$ piecewise NCP function and then define a $4-1$ piecewise NCP function:

$$
\Phi(a, b)=\left\{\begin{array}{lll}
3 a-\left(\frac{a^{2}}{b}\right), & b \geq a>0 & \text { or } \quad 3 b>-a \geq 0 ; \\
3 a-\left(\frac{b^{2}}{a}\right), & a>b>0 & \text { or } 3 a>-b \geq 0 ; \\
9 a+9 b, & \text { else. }
\end{array}\right.
$$

If $(a, b) \neq(0,0)$, then

$$
\nabla \Phi(a, b)=\left\{\begin{array}{l}
\left(\begin{array}{c}
3-\left(\frac{2 a}{b}\right) \\
\left(\frac{a^{2}}{b^{2}}\right)
\end{array}\right), \quad b \geq a>0, \text { or } 3 b>-a \geq 0 ; \\
\left(\frac{b^{2}}{a^{2}}\right) \\
3-\left(\frac{2 b}{a}\right) \\
\left(\begin{array}{c}
9 \\
9
\end{array}\right), \\
\text { else. }
\end{array}\right)
$$

We define the $4-1$ piecewise linear NCP function ( $k$ is any positive integer):

$$
\Phi(a, b)= \begin{cases}k^{2} a, & \text { if } \quad b \geq k|a| ; \\ 2 k b-\left(\frac{b^{2}}{a}\right), & \text { if } \quad a>\frac{|b|}{k} ; \\ 2 k^{2} a+2 k b+\left(\frac{b^{2}}{a}\right), & \text { if } \quad a<-\frac{|b|}{k} ; \\ k^{2} a+4 k b, & \text { if } \quad b \leq-k|a|<0 .\end{cases}
$$

If $(a, b) \neq(0,0)$, then 


$$
\nabla \Phi(a, b)= \begin{cases}\left(\begin{array}{c}
k^{2} \\
0
\end{array}\right), & \text { if } \quad b \geq k|a| ; \\
\left(\frac{b^{2}}{a^{2}}\right) & \\
\left.2 k-\left(\frac{2 b}{a}\right)\right), & \text { if } \quad a>\frac{|b|}{k} ; \\
\left(2 k^{2}-\left(\frac{b^{2}}{a^{2}}\right)\right. \\
2 k+\left(\frac{2 b}{a}\right) \\
\left(\begin{array}{c}
k^{2} \\
4 k
\end{array}\right), & \text { if } \quad a<-\frac{|b|}{k} ;\end{cases}
$$

Denote $H: R^{2 n} \longrightarrow R^{2 n}$,

$$
H(x, t)=\left(\begin{array}{c}
t-F(x) \\
\Phi(x, t)
\end{array}\right),
$$

where $t$ is a sequence in the algorithm and $t=F(x)$ holds at the optimal solution to NCP.

Hence, the NCP can be written as the following minimization problem:

$$
\min \Psi(x, t)=\|H(x, t)\| .
$$

To get the solution of (7), we introduce the notations as follows:

$$
\begin{aligned}
\left(\alpha_{i}^{k}, \beta_{i}^{k}\right) & = \begin{cases}(1,1), & (x, t)=(0,0) \\
\nabla \Phi(x, t), & \text { otherwise. }\end{cases} \\
i & =1,2, \ldots, n . \text { Obviously, } \alpha_{i}^{k}>0 \text { and } \beta_{i}^{k}>0 .
\end{aligned}
$$
get

Denote the Jacobian matrix of $H\left(x^{k}, t^{k}\right)$ by $V\left(x^{k}, t^{k}\right)$, we

$$
V\left(x^{k}, t^{k}\right)=\left(\begin{array}{cc}
-F \prime\left(x^{k}\right) & I \\
\operatorname{diag}\left(\alpha_{i}^{k}\right) & \operatorname{diag}\left(\beta_{i}^{k}\right)
\end{array}\right) .
$$

The identity matrix of $n \times n$, diagonal matrix whose $i$ th diagonal element is $\alpha_{i}^{k}$, and the diagonal matrix whose $i$ th diagonal element is $\beta_{i}^{k}$ are represented by $I, \operatorname{diag}\left(\alpha_{i}^{k}\right)$, and $\operatorname{diag}\left(\beta_{i}^{k}\right)$, respectively.

We use the nonmonotone line search to present Broyden-like method. The search directions $d$ and $\lambda$ are obtained by calculating a system of smooth equation, and the algorithm is described in detail in Algorithm1.

\section{Convergence Analysis}

In this section, the global convergence properties of a Broyden-like algorithm with 3-1 piecewise NCP function are discussed. We give some assumptions to prove the convergence of the algorithm.

\section{Assumption 1}

(a) Suppose $F: R^{n} \longrightarrow R^{n}$ is $P_{0}$-function and it is continuously differentiable.

(b) On the level set of

$$
L\left(x^{0}, t^{0}\right)=\left\{(x, t) \in R^{2 n} \mid \Psi t(x, t) n \leq q \Psi h\left(x^{0}, t^{0}\right)\right\},
$$

where $F$ is Lipschitz continuously differentiable, namely, there exists a constant $L$ such that for all $x_{1}, x_{2} \in R^{n}$,

$$
\left\|F\left(x_{1}\right)-F\left(x_{2}\right)\right\| \leq L\left\|x_{1}-x_{2}\right\| .
$$

Remark 1 (see [27]). $F(x)$ is $P_{0}$-function, then $F^{\prime}(x)$ is positive semidefinite.

Lemma 1. If $H\left(x^{0}, t^{0}\right) \neq 0$, then $B_{0}=V_{0}$ is nonsingular.

Proof. Assume $H\left(x^{0}, t^{0}\right) \neq 0$. If $V_{0}^{T}(u, v)=0$ for some $(u, v) \in R^{2 n}$, where $u=\left(u_{1}, u_{2}, \ldots, u_{n}\right)^{T}$ and $v=\left(v_{1}, v_{2}, \ldots\right.$, $\left.v_{n}\right)^{T}$, then

$$
\begin{aligned}
-F \prime\left(x^{0}\right) u+I v & =0, \\
\operatorname{diag}\left(\alpha^{0}\right) u+\operatorname{diag}\left(\beta^{0}\right) v & =0 .
\end{aligned}
$$

By the definitions of $\alpha_{i}^{0}$ and $\beta_{i}^{0}$, for all $i, \alpha_{i}^{0}>0$ and $\beta_{i}^{0}>0$. Therefore, $\operatorname{diag}\left(\beta^{0}\right)$ is nonsingular. Then

$$
v=-\left(\operatorname{diag}\left(\beta^{0}\right)\right)^{-1} \operatorname{diag}\left(\alpha^{0}\right) u .
$$

Substitute $v$ in (12) by (14), and multiply by $u^{T}$, we have

$$
-u^{T} F\left(x^{0}\right) u-u^{T}\left(\operatorname{diag}\left(\beta^{0}\right)\right)^{-1} \operatorname{diag}\left(\alpha^{0}\right) u=0 .
$$

According to the definition of $P_{0}$-function, all the principal minor determinants of $F_{\prime}(x)$ is nonnegative; hence, $F^{\prime}(x)$ is positive semidefinite. And matrix $\left(\operatorname{diag}\left(\beta^{0}\right)\right)^{-1} \operatorname{diag}\left(\alpha^{0}\right)$ is positive definite. Therefore $u=0$. Together with (14), it holds that $v=0$, which implies $B_{0}$ is nonsingular.

Lemma 2. Assume that Assumption 1 holds. Then $\Phi\left(x^{k}, t^{k}\right) \longrightarrow 0$, as $k \longrightarrow \infty$.

Proof. For convenience, we define $\left\|\Phi^{l(k)}\right\|=\max _{0 \leq r \leq m(k)-1} \|$ $\Phi^{k-r} \|$, where $\quad k-m(k)+1 \leq l(k) \leq k$. When $m(k+1) \leq m(k)+1$, we have 


$$
\begin{aligned}
\left\|\Phi^{l(k+1)}\right\| & =\max _{0 \leq r \leq m(k+1)-1}\left\|\Phi^{k+1-r}\right\| \\
& \leq \max _{0 \leq r \leq m(k)}\left\|\Phi^{k+1-r}\right\| \\
& =\max \left\{\left\|\Phi^{l(k)}\right\|,\left\|\Phi^{k+1}\right\|\right\} \\
& =\left\|\Phi^{l(k)}\right\| .
\end{aligned}
$$

Which means $\left\|\Phi^{l(k)}\right\|$ is decreasing monotonely, and hence, we have $\left\{\left\|\Phi^{l(k)}\right\|\right\}$ convergent. Based on (c) of Algorithm1, we have $\left\|\Phi^{l(k)}\right\| \leq\left\|\Phi^{l(l(k)-1)}\right\|$.

By $\xi \in(0,1), \quad\left\{\left\|\Phi^{l(k)}\right\|\right\} \longrightarrow 0(k \longrightarrow \infty)$ holds, so according to $\left\|\Phi^{k+1}\right\| \leq \xi\left\|\Phi^{l(k)}\right\| \longrightarrow 0$, the conclusion holds.

Lemma 3. Assume Assumption 1 holds. Then $t^{k}-F\left(x^{k}\right) \longrightarrow 0$ as $k \longrightarrow \infty$.

Proof. Define $\left\|t^{l(k)}-F\left(x^{l(k)}\right)\right\|=\max _{0 \leq r \leq m(k)-1} \| t^{k-r_{-}}$ $F\left(x^{k-r}\right) \|$, where $k-M \leq l(k) \leq k$. For $m(k+1) \leq m(k)+1$, we have

$$
\begin{aligned}
\left\|t^{l(k+1)}-F\left(x^{l(k+1)}\right)\right\| & =\max _{0 \leq r \leq m(k+1)-1}\left\|t^{k+1-r}-F\left(x^{k+1-r}\right)\right\| \\
& \leq \max _{0 \leq r \leq m(k)}\left\|t^{k+1-r}-F\left(x^{k+1-r}\right)\right\| \\
& =\max \left\{\left\|t^{l(k)}-F\left(x^{l(k)}\right)\right\|,\left\|t^{k+1}-F\left(x^{k+1}\right)\right\|\right\} \\
& =\left\|t^{l(k)}-F\left(x^{l(k)}\right)\right\| .
\end{aligned}
$$

From (17), $\left\|t^{l(k)}-F\left(x^{l(k)}\right)\right\|$ is decreasing in a monotone way; then $\left\{\left\|t^{l(k)}-F\left(x^{l(k)}\right)\right\|\right\}$ is convergent.

According to (g) of Algorithm 1, $\left\|t^{l(k)}-F\left(x^{l(k)}\right)\right\|$ $\leq \xi\left\|t^{l(l(k)-10)}-F\left(x^{l(l(k)-1)}\right)\right\|$. By $\quad \xi \in(0,1), \quad\left\{\| t^{l(k)}-\right.$ $\left.F\left(x^{l(k)}\right) \|\right\} \longrightarrow 0(k \longrightarrow \infty)$ holds. That means $\left\|t^{k+1}-F\left(x^{k+1}\right)\right\| \leq \xi\left\|t^{l(k)}-F\left(x^{l(k)}\right)\right\| \longrightarrow 0$ holds by Algorithm1, so the conclusion is as follows.

Lemma 4. Assume Assumption 1 holds. Then $d^{k} \longrightarrow 0$, $\lambda^{k} \longrightarrow 0$, and $H^{k} \longrightarrow 0$, as $k \longrightarrow \infty$.

Proof. We have $\Phi\left(x^{k}, t^{k}\right) \longrightarrow 0,\left[t^{k}-F\left(x^{k}\right)\right] \longrightarrow 0$, as $k \longrightarrow \infty$ by Lemma 2 and Lemma 3 .

So, $H\left(x^{k}, t^{k}\right) \longrightarrow 0$, as $k \longrightarrow \infty$ :

$$
B_{k}\left(\begin{array}{l}
d^{k} \\
\lambda^{k}
\end{array}\right)=\left(\begin{array}{c}
F\left(x^{k}\right)-t^{k} \\
-\Phi\left(x^{k}, t^{k}\right)
\end{array}\right)=0 .
$$

We know that $B_{k}$ is nonsingular by Algorithm1. So, $d^{k} \longrightarrow 0$, and $\lambda^{k} \longrightarrow 0$, as $k \longrightarrow \infty$.

Theorem 1. Under the same condition in Lemma 4, equation (a) of Algorithm1 has solutions, and the definition of Algorithm1 is well.
Proof. On the one hand, we know $B_{0}$ is nonsingular by Lemma 1 . And $B_{k}$ produced by the Broyden-like iteration is nonsingular. Hence equation (a) of Algorithm1 has one and only one solution. On the other hand, we know $\Phi\left(x^{k}, t^{k}\right) \longrightarrow 0$ and $\left[t^{k}-F\left(x^{k}\right)\right] \longrightarrow 0$ as $k \longrightarrow \infty$ by Lemma 2 and Lemma 3. So $\left\|H\left(x^{k}, t^{k}\right)\right\| \longrightarrow 0$ as $k \longrightarrow \infty$.

Lemma 5. Assume Assumption 1 holds, and let $\left\{\left(x^{k}, t^{k}\right)\right\}$ be generated sequence by Algorithm 1 ; then $\left\{\left(x^{k}, t^{k}\right)\right\} \subset L\left(x^{0}, t^{0}\right)$.

Proof. By induction, for $k=0$, we have $\left(x^{0}, t^{0}\right) \in L\left(x^{0}, t^{0}\right)$. Assume $\left(x^{k}, t^{k}\right) \in L\left(x^{0}, t^{0}\right)$; then we have $\Psi\left(x^{k}, t^{k}\right) \leq \Psi\left(x^{0}, t^{0}\right)$. By (c) and (d) of Algorithm1, we get

$$
\begin{aligned}
\Psi\left(x^{k+1}, t^{k+1}\right) & =\left\|\Phi\left(x^{k+1}, t^{k+1}\right)\right\|+\left\|t^{k+1}-F\left(x^{k+1}\right)\right\| \\
& \leq \xi \max _{0 \leq r \leq m(k)-1}\left(\left\|\Phi^{k-r}\right\|+\left\|t^{k-r}-F\left(x^{k-r}\right)\right\|\right) \\
& =\xi \max _{0 \leq r \leq m(k)-1} \Psi\left(x^{k-r}, t^{k-r}\right) \\
& \leq \Psi\left(x^{0}, t^{0}\right) .
\end{aligned}
$$

So, $\left(x^{k+1}, t^{k+1}\right) \in L\left(x^{0}, t^{0}\right)$. Based on the similar analysis, it is easy to see $\left\{\left(x^{k}, t^{k}\right)\right\} \subset L\left(x^{0}, t^{0}\right)$ for all $k$.

Theorem 2. Assume Assumption 1 holds, and $\left\{\left(x^{k}, t^{k}\right)\right\}$ is generated by Algorithm1; then there exists an accumulation point $\left(x^{*}, t^{*}\right)$ of the sequence $\left\{\left(x^{k}, t^{k}\right)\right\}$ which is solution of $N C P(1)$.

Proof. From Lemma 3 and Lemma 4, we know $\left\{\left(x^{k}, t^{k}\right)\right\} \subset L\left(x^{0}, t^{0}\right)$. By Assumption 1(b), we see that $L\left(x^{0}, t^{0}\right)$ is bounded. So, $\left\{\left(x^{k}, t^{k}\right)\right\}$ has an accumulation point. Suppose there exists a subsequence $\left\{\left(x^{k}, t^{k}\right)\right\}_{k \in K}$ which has an accumulation point $\left(x^{*}, t^{*}\right)$. We should prove $H\left(x^{*}, t^{*}\right)=0$.

Suppose $\left\{\left(x^{k}, t^{k}\right)\right\}_{k \in K}$ be an infinite sequence generated by Algorithm1. By construction of the algorithm, we know there are two types of successive iteration. Let $K_{1}=\left\{k \mid x^{k+1}=x^{k}+d^{k}, t^{k+1}=t^{k}+\lambda^{k}\right\}$ and $K_{2}=\left\{k \mid x^{k+1}=\right.$ $\left.x^{k}+\rho_{k} d^{k}, t^{k+1}=t^{k}+\rho_{k} \lambda^{k}\right\}$. We need to prove the conclusion by the following two cases:

Case I: $K_{1}$ is an infinite index set. Let the sequence be $\left\{\left(x^{k}, t^{k}\right)\right\}_{k \in K_{1}}$, which satisfy (b) of Algorithm 1 . Therefore,

$$
\Psi^{k_{1}} \leq \xi \Psi^{k_{2}} \leq \xi^{2} \Psi^{k_{3}} \leq \cdots \leq \xi^{m-1} \Psi^{k_{m}}
$$

This suggests that $\liminf _{k \rightarrow \infty} H\left(x^{k}, t^{k}\right)=0$.

Case II: $K_{2}$ is an infinite index set. Let the sequence be $\left\{\left(x^{k}, t^{k}\right)\right\}_{k \in K_{2}}$, which satisfy (f) and (g) of Algorithm 1 .

It is known that $\left\|\Phi^{l(k)}\right\|$ is monotone decreasing and $\lim _{k \longrightarrow \infty}\left\|\Phi^{k}\right\|=0$ by Lemma 2 and $\left\|t^{l(k)}-F\left(x^{l(k)}\right)\right\|$ is 
Step 0: initialization.

Given initial point $\left(x^{0}, t^{0}\right) \in R^{2 n}, \mu \in(0,1), \xi>0, \bar{\xi}<1, B_{0}=V\left(x^{0}, t^{0}\right), k=0$.

Step 1: if $\Psi\left(x^{k}, t^{k}\right)=0$, then stop. Otherwise, calculate the search direction

$B_{k}\left(\begin{array}{l}d \\ \lambda\end{array}\right)=\left(\begin{array}{c}F\left(x^{k}\right)-t^{k} \\ -\Phi\left(x^{k}, t^{k}\right)\end{array}\right) \cdot(\mathrm{a})$

By (a), we can obtain $d^{k}$ and $\lambda^{k}$.

Step 2: modified linear search technique.

Step 2.1 If

$$
\begin{aligned}
& \Psi\left(x^{k}+d^{k}, t^{k}+\lambda^{k}\right) \leq \xi \Psi\left(x^{k}, t^{k}\right),(\mathrm{b}) \\
& \left\|\Psi\left(x^{k}+d^{k}, t^{k}+\lambda^{k}\right)\right\| \leq \xi \max _{0 \leq r \leq m(k)-1}\left\|\Psi\left(x^{k-r}, t^{k-r}\right)\right\|,(\mathrm{c}) \\
& \left\|t^{k}+\lambda^{k}-F\left(x^{k}+d^{k}\right)\right\| \leq \xi \max _{0 \leq r \leq m(k)-1}\left\|t^{k-r}-F\left(x^{k-r}\right)\right\|,(\mathrm{d})
\end{aligned}
$$

where $m(0)=0,0 \leq m(k) \leq \min \{m(k-1)+1, M\}$ is a positive constant. Then, let $x^{k+1}=x^{k}+d^{k}, t^{k+1}=t^{k}+\lambda^{k}$, (e)

and go to Step 3; otherwise, go to Step 2.2.

Step 2.2: for $j=0,1, \ldots$, check the following inequality with $\mu^{j}$ successively

$\left\|\Phi\left(x^{k}+\mu^{j} d^{k}, t^{k}+\mu^{j} \lambda^{k}\right)\right\| \leq \xi \max _{0 \leq r \leq m(k)-1}\left\|\Phi\left(x^{k-r}, x^{k-r}\right)\right\|$, (f)

$\left\|s^{k}+\mu^{j} \lambda^{k}-F\left(x^{k}+\mu^{j} d^{k}\right)\right\| \leq \xi \max _{0 \leq r \leq m(k)-1}\left\|t^{k-r}-F\left(x^{k-r}\right)\right\|$, (g)

Let $j_{k}$ be the smallest nonnegative integer $j$ such that (f) and (g) hold for $\mu^{j}$. Set $\rho_{k}$ : $=\mu^{j_{k}}$, and $x^{k+1}=x^{k}+\rho_{k} d^{k}, t^{k+1}=t^{k}+\rho_{k} \lambda^{k},(\mathrm{~h})$

and go to Step 3 .

Step 3: Update $B_{k}$ to get $B_{k+1}$,

$$
B_{k+1}=B_{k}+\xi_{k}\left(\left(z^{k}\right)^{T}\left(y^{k}-B_{k} z^{k}\right) /\left\|z^{k}\right\|^{2}\right) \text {, (i) }
$$

where

$$
z^{k}=\left(\begin{array}{c}
x^{k+1} \\
t^{k+1}
\end{array}\right)-\left(\begin{array}{c}
x^{k} \\
t^{k}
\end{array}\right), y^{k}=H\left(x^{k+1}, t^{k+1}\right)-H\left(x^{k}, t^{k}\right) \text {. (j) }
$$

Select $\xi_{k}$ to satisfy $\left|\xi_{k}-1\right| \leq \bar{\xi}$ and matrix $B_{k+1}$ is nonsingular.

Step 4: Let $k=k+1$, go to Step 1 .

Algorithm 1

monotone decreasing and $\lim _{k \rightarrow \infty}\left\|t^{k}-F\left(x^{k}\right)\right\|=0$ by Lemma 3. Therefore, $\lim _{k \longrightarrow \infty}\left\|H\left(x^{k}, t^{k}\right)\right\|=\lim _{k \longrightarrow \infty}\left\|\Phi^{k}\right\|+$ $\lim _{k \rightarrow \infty}\left\|t^{k}-F\left(x^{k}\right)\right\|=0$ as $k \in K_{2}$.

Therefore, the conclusion is followed.

\section{Numerical Results}

In this section, some numerical results are given. We used a personal computer with $4.0 \mathrm{~GB}$ memory and Intel(R) Core(TM)i5-5200U CPU @2.20 GHz to perform all experiments. We used Windows 10 as the operating system and Matlab R2018b to write the computer codes. In the whole experiment, the parameters used in Algorithm 1 were $\xi=0.9, \mu=0.8, \xi_{k} \equiv 1, M$ is an integer which is randomly selected from 2 to 5 . $\|H(x, t)\|<10^{-6}$ was the stop criterion.

The number of iterations, the CPU time in seconds, and the value of $x^{(T)} F(x)$ at the final iteration are are listed in Table $1 . x_{0}$ in Table 1 means the Initial point where ones $(i, 1)$ means the $i$ dimension of this problem.

4.1. Some Test Problems. Examples 1-8 (NCP) are considered.

Example 1. Consider (1), where $x \in R^{n}$ and $F(x)=M x+q$ with

$$
M=\left(\begin{array}{ccccc}
4 & -1 & & & \\
-1 & 4 & -1 & & \\
& \ddots & \ddots & \ddots & \\
& & \ddots & \ddots & -1 \\
& & & -1 & 4
\end{array}\right),
$$

$$
q=\left(\begin{array}{c}
-1 \\
-1 \\
\vdots \\
-1 \\
-1
\end{array}\right)
$$

We use $x^{0}=(1,1, \ldots, 1)^{T}$ and $t^{0}=\left(10^{-3}, 10^{-3}\right.$ $\left., \ldots, 10^{-3}\right)^{T}$ as the starting points to text this problem.

Example 2. Consider (1), where $x \in R^{3}$, and $F(x): R^{3} \longrightarrow R^{3}$ given by

$$
F(x)=\left(\begin{array}{c}
x_{2} \\
x_{3} \\
-x_{2}+x_{3}+1
\end{array}\right) \text {. }
$$

Figure 1 is the $3 \mathrm{D}$ diagram of Example 2. $(0, \lambda, 0)$ is an infinite solution of this problem, where $\lambda \in[0,1]$. The initial points $x^{0}, t^{0}$ are randomly generated, and these elements are in the interval $(0,10)$. 
Example 3. Consider (1), where $x \in R^{7}$, and $F(x): R^{7} \longrightarrow R^{7}$ given by

$$
F(x)=\left(\begin{array}{c}
2 x_{1}-x_{3}+x_{5}+3 x_{6}-1 \\
x_{2}+2 x_{5}+x_{6}-x_{7}-3 \\
-x_{1}+2 x_{3}+x_{4}+x_{5}+2 x_{6}-4 x_{7}+1 \\
x_{3}+x_{4}+x_{5}-x_{6}-1 \\
-x_{1}-2 x_{2}-x_{3}-x_{4}+5 \\
-3 x_{1}-x_{2}-2 x_{3}+x_{4}+4 \\
x_{2}+4 x_{3}-1.5
\end{array}\right) .
$$

Example 4. Consider (1), where $x \in R^{4}$ and $F(x): R^{4} \longrightarrow R^{4}$ given by

$$
F(x)=\left(\begin{array}{c}
x_{1}^{3}-8 \\
x_{2}+x_{2}^{3}-x_{3}+3 \\
x_{2}+x_{3}+2 x_{3}^{3}-3 \\
x_{4}+2 x_{4}^{3}
\end{array}\right)
$$

Example 5 (Kojima-Shindo Problem). Consider (1), where $x \in R^{4}$ and $F(x): R^{4} \longrightarrow R^{4}$ given by

$$
F(x)=\left(\begin{array}{c}
3 x_{1}^{2}+2 x_{1} x_{2}+2 x_{2}^{2}+x_{3}+3 x_{4}-6 \\
2 x_{1}^{2}+x_{1}+x_{2}^{2}+10 x_{3}+2 x_{4}-2 \\
3 x_{1}^{2}+x_{1} x_{2}+2 x_{2}^{2}+2 x_{3}+9 x_{4}-9 \\
x_{1}^{2}+3 x_{2}^{2}+2 x_{3}+3 x_{4}-3
\end{array}\right) .
$$

$\left(\sqrt{6} / 2, t n 0 q, h_{0} x, 7 C 0.5\right)$ is a degenerate solution, and $(1,0,3,0)$ is a nondegenerate solution.

Example 6 (Modified Mathiesen Problem). Consider (1), where $x \in R^{4}$ and $F(x): R^{4} \longrightarrow R^{4}$ given by

$$
F(x)=\left(\begin{array}{c}
-x_{2}+x_{3}+x_{4} \\
x_{1}-\frac{4.5 x_{3}+2.7 x_{4}}{x_{2}+1} \\
5-x_{1}-\frac{0.5 x_{3}+0.3 x_{4}}{x_{3}+1} \\
3-x_{1}
\end{array}\right)
$$

Example 7. The function $f(x)$ is endowed with the component as follows:

$$
\begin{aligned}
& F(x)=\left(f_{1}(x), f_{2}(x), \ldots, f_{n}(x)\right)^{T} \\
& f_{i}(x)=e^{x_{i}}-1, \quad i=1,2, \ldots, n-1, \\
& f_{n}(x)=e^{x_{n}}+x_{n}-1 .
\end{aligned}
$$

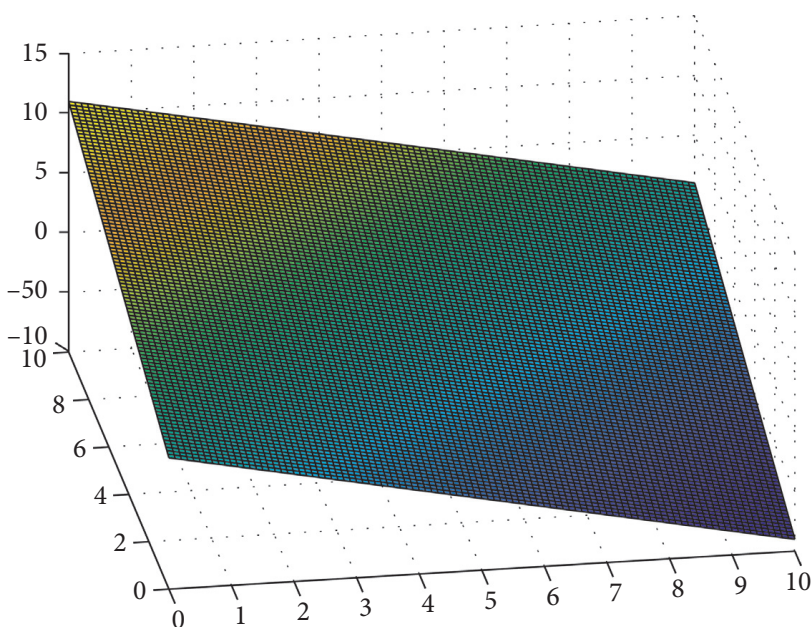

Figure 1: Diagram of Example 2.

Example 8. Consider (1), where $x \in R^{n}$ and $F(x)=M x+q$ with

$$
M=\operatorname{diag}\left(\frac{1}{n}, \frac{2}{n}, \ldots, 1\right), q=(-1,-1, \ldots,-1)^{T} .
$$

Table 1 shows the results of Examples 1-8 using 3-1 piecewise, 4-1 piecewise Algorithm1 and feasible direction method, respectively. It can be seen from the table that Algorithm1 applying 3-1 piecewise has a good solution to all the above problems. Algorithm1 applying 4-1 piecewise is slightly insufficient, and the feasible direction method has some difficulties in solving examples above, and some of the examples cannot be solved. Figure 2 shows how the $x^{T} f(x)$ value of the three algorithms decreases as the number of iterations increases in each specific example. We use performance profiles [28]-distribution functions for a performance metric-as a tool for comparing different algorithms. We consider the comprehensive performance of the above three algorithms in terms of CPU time, number of iterations, and $x^{T} f(x)$ value. If the curve is closer to 1 , the better the ability to solve the problem (Figure 3).

4.2. Nash Equilibrium Problem. General economic equilibrium [29] means that total supply and total demand are exactly equal in a price system. With the existing productivity and technical conditions, producers get the most profit, while consumers get the most utility when they meet the budget constraints. The theory of general economic equilibrium was first put forward by the French economist Walras. Walras believes that when the whole economy is in equilibrium, the prices of all consumer goods and factors of production will have a certain equilibrium value, and their output and supply will have a certain equilibrium quantity. It is assumed that the whole economic system is a large and complete trading market, and the equilibrium price system means that all commodities are traded in this market, and finally all commodities can be traded. 
TABle 1: Iterations, CPU time, and $x^{T} f(x)$ for NCP Examples 2-6 between Algorithm1 and FDA.

\begin{tabular}{|c|c|c|c|c|c|c|c|c|c|c|}
\hline \multirow{2}{*}{ Problem } & \multirow{2}{*}{$x^{0}$} & \multicolumn{3}{|c|}{ Algorithm 1 with $3-1$ piecewise } & \multicolumn{3}{|c|}{ Algorithm 1 with $4-1$ piecewise } & \multicolumn{3}{|c|}{ Feasible directions algorithm } \\
\hline & & Iter & CPU time & $x^{T} f(x)$ & Iter & CPU time & $x^{T} f(x)$ & Iter & CPU time & $x^{T} f(x)$ \\
\hline \multirow{5}{*}{4.1} & \multirow{2}{*}{ ones $(100,1)$} & 2 & 0.000814 & $5.49 E-09$ & 13 & 0.001768 & $1.12 E-08$ & 5 & 0.004301 & $6.58 E-07$ \\
\hline & & 2 & 0.473808 & $5.69 E-08$ & 13 & 1.520458 & $1.21 E-08$ & 5 & 0.257754 & $-8.81 E-14$ \\
\hline & ones $(4069,1)$ & 3 & 15.640096 & $-1.58 E-11$ & 13 & 18.551757 & $1.16 E-08$ & 5 & 8.556959 & $7.20 E-14$ \\
\hline & ones $(8138,1)$ & 2 & 51.204458 & $4.51 E-10$ & 13 & 70.145272 & $1.44 E-08$ & 5 & 53.324527 & $-1.27 E-12$ \\
\hline & $(1,1,1)^{T}$ & 3 & 0.000235 & $-1.02 E-07$ & 8 & 0.000617 & $-5.41 E-08$ & 10 & 0.000354 & $4.66 E-07$ \\
\hline \multirow{3}{*}{4.2} & $(10,10,10)^{T}$ & 4 & 0.000288 & $5.25 E-08$ & 10 & 0.000604 & $3.26 E-07$ & 13 & 0.000529 & $3.76 E-07$ \\
\hline & $(1,5,9)^{T}$ & 7 & 0.000462 & $-2.23 E-08$ & 5 & 0.000742 & $4.64 E-07$ & 19 & 0.001465 & $3.50 E-07$ \\
\hline & ones $(7,1)$ & 15 & 0.001014 & $-2.23 E-08$ & 22 & 0.001768 & $5.53 E-07$ & 26 & 0.004301 & $6.58 E-07$ \\
\hline \multirow{2}{*}{4.3} & $10 *$ ones $(7,1)$ & 15 & 0.001661 & $-4.62 E-09$ & 24 & 0.002366 & $1.05 E-08$ & $\mathrm{NaN}$ & $\mathrm{NaN}$ & $\mathrm{NaN}$ \\
\hline & $10 * \operatorname{rand}(7,1)$ & 16 & 0.001608 & $-2.14 E-09$ & 20 & 0.001204 & $1.20 E-08$ & $\mathrm{NaN}$ & $\mathrm{NaN}$ & $\mathrm{NaN}$ \\
\hline \multirow{2}{*}{4.4} & $(1,1,1,1)^{T}$ & 14 & 0.007181 & $-3.11 E-08$ & 23 & 0.001696 & $-6.07 E-07$ & $>500 *$ & Inf & $\mathrm{NaN}$ \\
\hline & $(10,10,10,10)^{T}$ & 145 & 0.019268 & $1.80 E-08$ & 86 & 0.004996 & $-2.26 E-07$ & 21 & 0.007137 & $6.12 E-07$ \\
\hline \multirow{2}{*}{4.5} & $(1,1,1,1)^{T}$ & 13 & 0.00103 & $-5.62 E-09$ & 26 & 0.001658 & $4.93 E-09$ & 36 & 0.001121 & $6.43 E-07$ \\
\hline & $(1,2,3,4)^{T}$ & 23 & 0.002497 & $-5.65 E-07$ & 30 & 0.003223 & $-2.75 E-08$ & 65 & 0.009764 & $9.61 E-07$ \\
\hline \multirow[t]{3}{*}{4.6} & $(1,1,1,1)^{T}$ & 10 & 0.001351 & $1.52 E-08$ & 9 & 0.001473 & $6.19 E-09$ & 16 & 0.00746 & $3.67 E-07$ \\
\hline & ones $(100,1)$ & 11 & 0.039089 & $-9.29 E-11$ & 17 & 0.001768 & $8.98 E-08$ & 46 & 0.024301 & $8.81 E-07$ \\
\hline & ones $(1024,1)$ & 20 & 1.230144 & $2.26 E-10$ & 20 & 1.745354 & $-2.68 E-11$ & 52 & 1.157443 & $7.57 E-07$ \\
\hline \multirow[t]{4}{*}{4.7} & ones $(4069,1)$ & 15 & 25.532848 & $-2.61 E-12$ & 20 & 35.615797 & $3.61 E-10$ & 55 & 50.806954 & $8.14 E-07$ \\
\hline & ones $(8138,1)$ & 15 & 305.640096 & $-4.09 E-14$ & 20 & 370.126146 & $4.62 E-09$ & 57 & 415.720027 & $7.56 E-07$ \\
\hline & ones $(100,1)$ & 4 & 0.002064 & $7.41 E-07$ & 57 & 0.107685 & $1.32 E-07$ & $\mathrm{NaN}$ & $\mathrm{NaN}$ & $\mathrm{NaN}$ \\
\hline & ones $(1024,1)$ & 6 & 0.641349 & $5.40 E-07$ & 407 & 22.002366 & $3.10 E-06$ & $\mathrm{NaN}$ & $\mathrm{NaN}$ & $\mathrm{NaN}$ \\
\hline \multirow[t]{2}{*}{4.8} & ones $(4069,1)$ & 8 & 26.727794 & $1.87 E-06$ & $>500^{*}$ & Inf & $\mathrm{NaN}$ & $\mathrm{NaN}$ & $\mathrm{NaN}$ & $\mathrm{NaN}$ \\
\hline & ones $(8138,1)$ & 10 & 215.640096 & $5.19 E-06$ & $>500^{*}$ & Inf & $\mathrm{NaN}$ & $\mathrm{NaN}$ & $\mathrm{NaN}$ & $\mathrm{NaN}$ \\
\hline
\end{tabular}

Considering the competitive economic model of production and investment, suppose $H$ is a price system, in which there are $N$ kinds of commodities, we use $R^{N}$ to express commodity space. For producer $i$, the set of production is $Y_{i} \subseteq R^{N}$. For consumer $j$, the set of consumption is $Z_{j} \subseteq R^{N}$. The number of producers and consumers in the system are $l$ and $k$, respectively. The total production, total consumption, and initial commodity reserve are represented by $Y_{i}, Z_{j}$, and $\lambda_{j}$, respectively, and the proportion of consumer $j$ in the profit of producer $i$ is represented by $\phi_{j i}$. Specially, $i=1, \ldots, l ; j=1, \ldots, k$; and $Z_{j}, Y_{i}, \lambda_{j} \in R^{N}$.

To describe the model better, we assume the following definitions. In particular, $Z_{j}, Y_{i}$, and $\lambda_{j}$ are independent of $x$.

Definition 1. Let $z_{j} \in Z_{j}, y_{i} \in Y_{i}, x$ is the equilibrium price:

(1) For every $i$, the maximum profit function is $x \cdot y_{i}$.

(2) For every $j$, preference maximum element is $z_{j}=$ $\left\{z_{j} \in Z_{j} \mid x t \cdot n z_{j} q \leq h x \cdot x \lambda_{j} 7+C \sum_{j=1}^{l} \phi_{j i} \cdot x \cdot y_{i}\right.$.

(3) Economic equilibrium is defined as $\sum_{i=1}^{l} \lambda_{i}+\sum_{i=1}^{l} x \cdot y_{i}-\sum_{j=1}^{k} z_{j}=0$.

It can be seen from Definition 1 that when price system $H$ reaches economic equilibrium, the demands of both producers and consumers are satisfied and then all the commodities of price system $H$ are sold, that is, the commodities are cleared. We define the conditions for clearing the goods as

$F=\sum_{i=1}^{l} \lambda_{i}+\sum_{i=1}^{l} x \cdot y_{i}-\sum_{j=1}^{k} z_{j}, \quad x \geq 0, x \cdot F=0$.
Equation (29) is not only the equilibrium state of free allocation, but also the model of linear complementarity problem. If $Z_{j}, Y_{i}$, and $\lambda_{j}$ are related to $x$, (29) will become a nonlinear complementarity problem (NCP).

Let the inverse demand function for the market be defined by

$$
P(Q)=5000^{(1 / \gamma)} Q^{-(1 / \gamma),}
$$

where $Q$ is the total quantity produced, $P$ is the market price, and $\gamma$ is the elasticity of demand with respect to price. Let $q_{i}$ denote the output of firm $i$ and let the total cost function for firm $i$ be given by

$$
\begin{aligned}
f_{i}\left(q_{i}\right) & =c_{i} q_{i}+\left(\frac{\beta_{i}}{1+\beta_{i}}\right) L^{\left(1 / \beta_{i}\right)} q_{i}^{\left(\left(\beta_{i}+1\right) / \beta_{i}\right)}, \\
F_{i}(q) & =f_{i}^{\prime}\left(q_{i}\right)-p \sum_{j=1}^{n} q_{j}-q_{i} p^{\prime} \sum_{j=1}^{n} q_{j}, \quad i=1,2, \ldots, n, \\
F & =\left[F_{1}(q), F_{2}(q), \ldots, F_{i}(q)\right] .
\end{aligned}
$$

Example 9. Data is given in Table 2.

Example 10. Data is given in Table 3.

4.3. Two-Dimensional Contact Problem. Under the conditions of nonpenetration and negligible attraction between objects, the elastic contact problem mainly requires the 

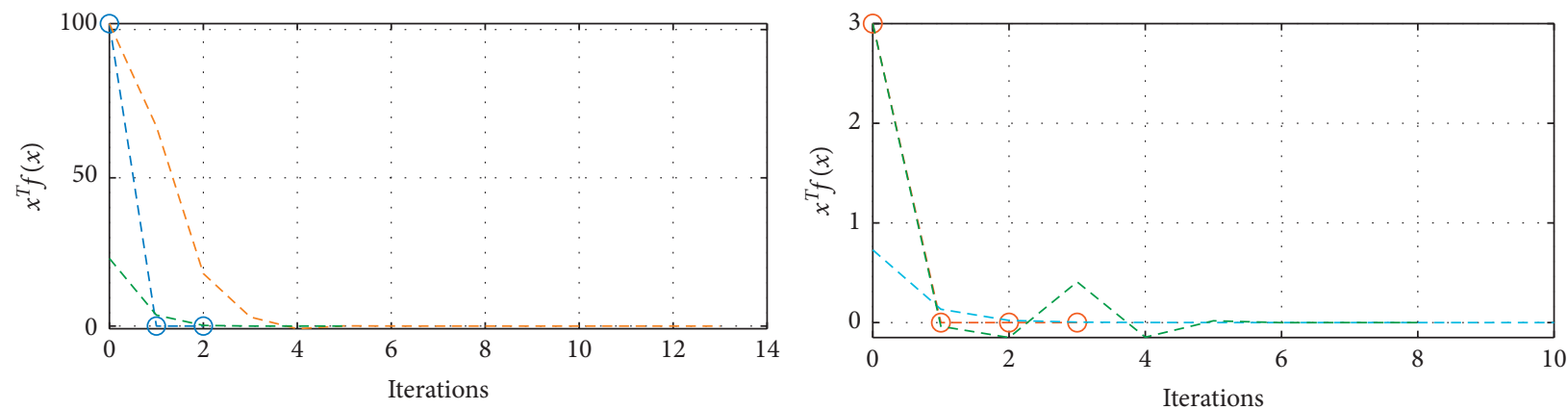

- $\theta-\operatorname{Alg} 2.1(3-1)$

- - Alg 2.1(4-1)

- - FDA

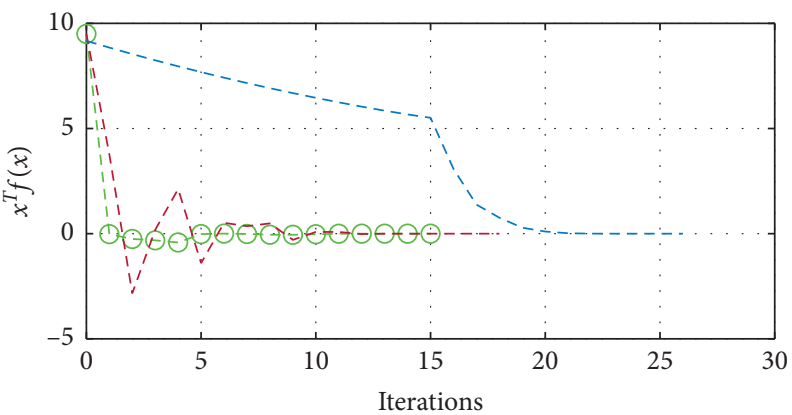

- $\theta-\operatorname{Alg} 2.1(3-1)$

- - FDA

- - $\operatorname{Alg} 2.1(4-1)$

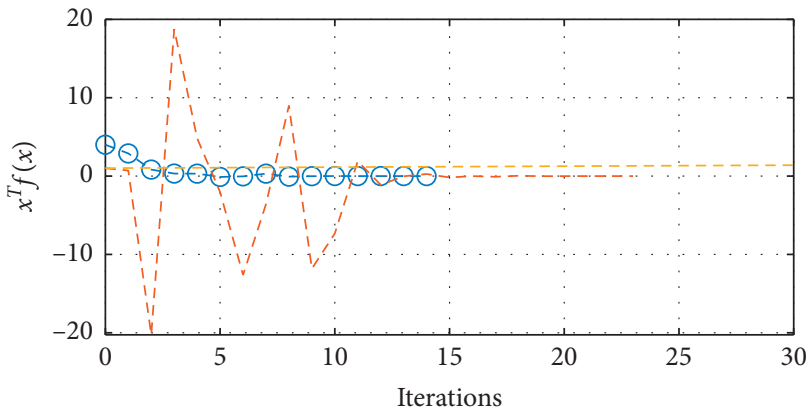

- $\theta-\operatorname{Alg} 2.1(3-1)$

- - Alg 2.1(4-1)

- . - FDA

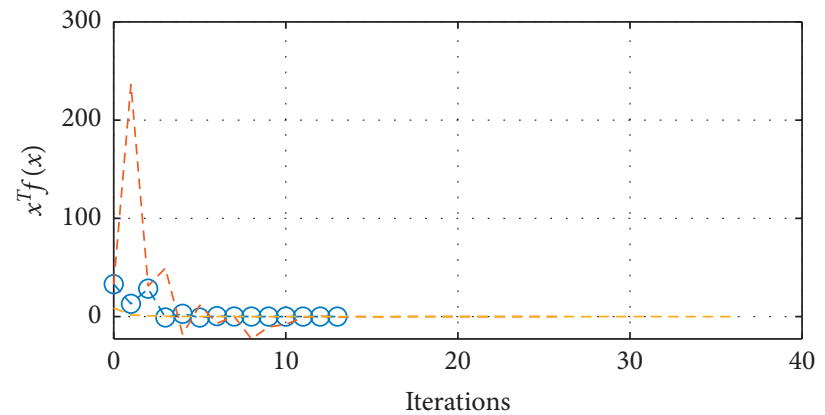

- $\theta-\operatorname{Alg} 2.1(3-1)$

- - Alg 2.1(4-1)

- - FDA

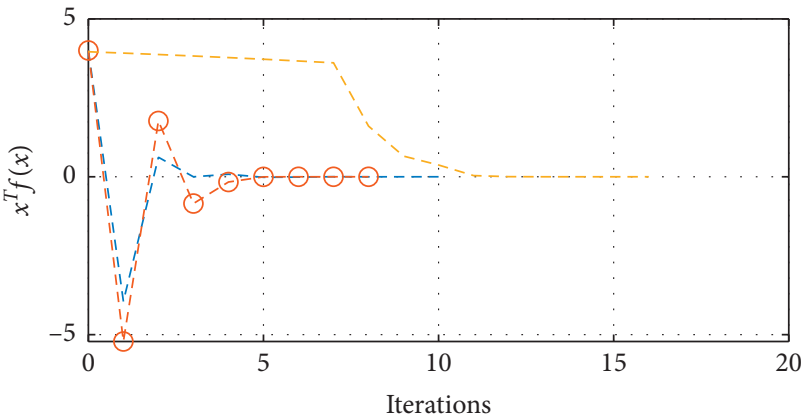

- $\theta-\operatorname{Alg} 2.1(3-1)$

- - Alg 2.1(4-1)

- . FDA

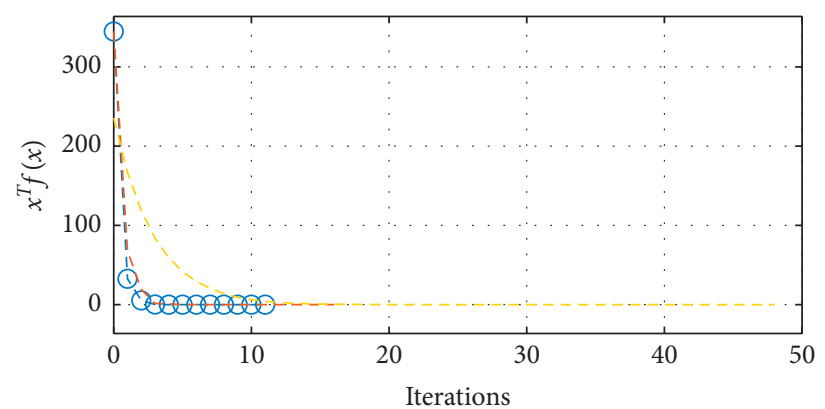

- - Alg 2.1(3-1)

$-\ominus-\operatorname{Alg} 2.1(4-1)$

- - FDA

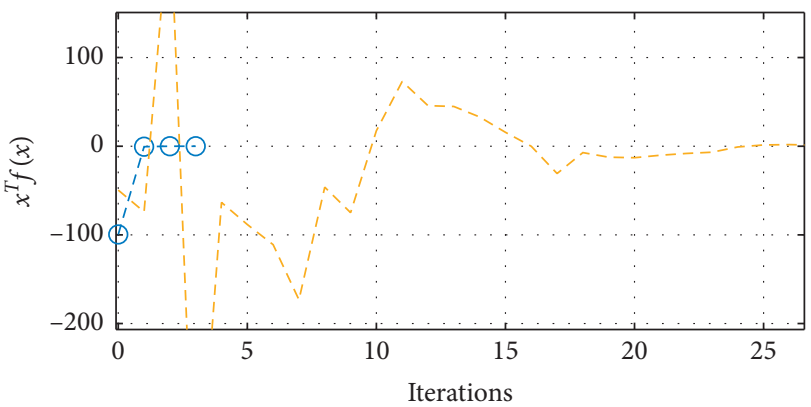

- - Alg 2.1(3-1)
- - Alg 2.1(4-1)
- - FDA

- $\theta-\operatorname{Alg} 2.1(3-1)$

- - Alg 2.1(4-1)

FIgURE 2: Schematic diagram of the changes of $x^{T} f(x)$ with iteration of the three algorithms (the same initial point of ones $(n, 1)$ ). 


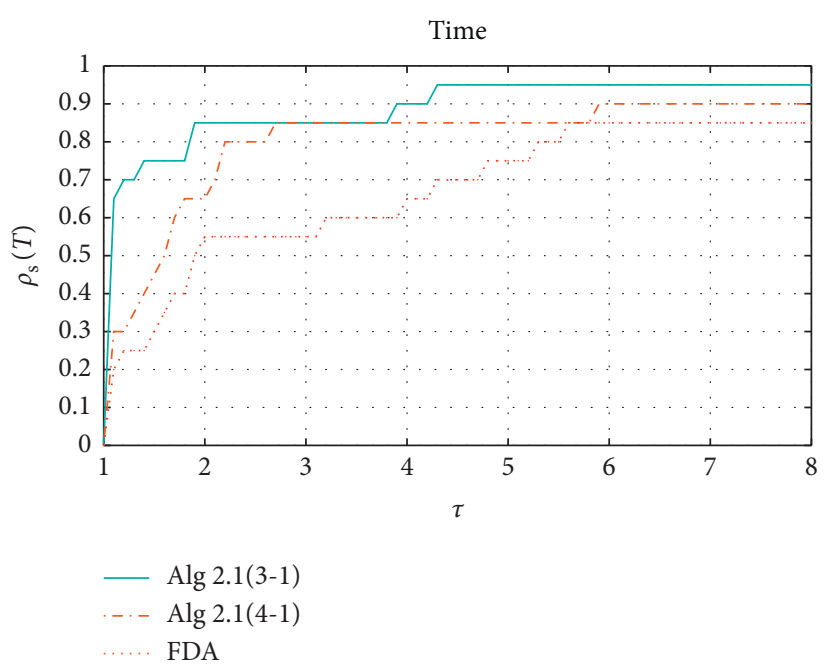

(a)

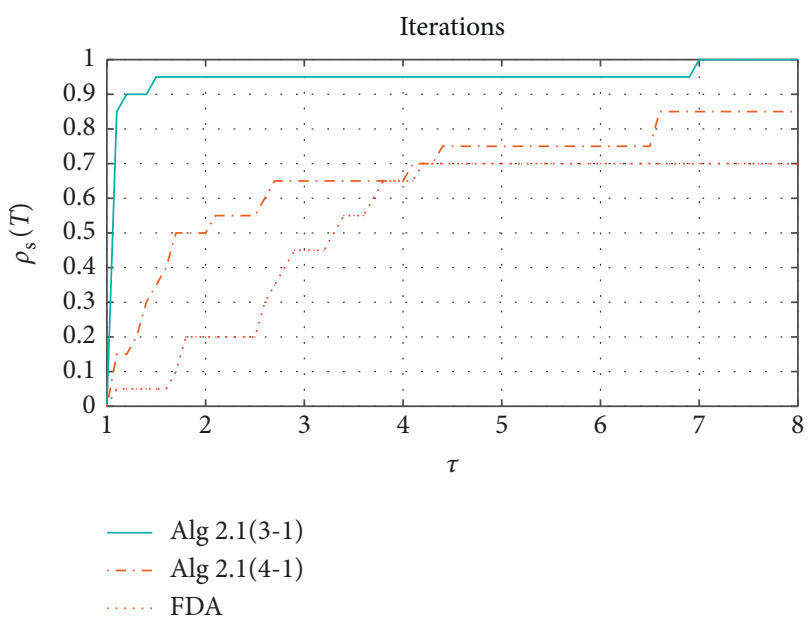

(b)

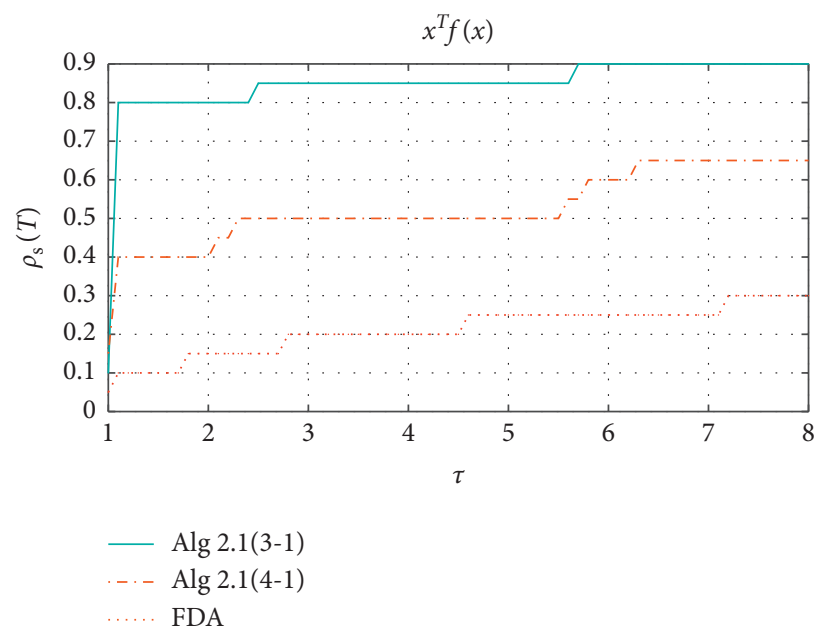

(c)

Figure 3: Performance profile for Algorithm1 and feasible directions algorithm through Examples 1-8.

contact surface and the pressure of the contact surface when two objects are pressed together. The wheel-rail problem is a typical elastic contact problem.

Figure 4 [31] shows the geometric structure application of the wheel-rail contact phenomenon, where Figure 4(a) represents the overall geometric structure showing the forward speed $\mathrm{V}$ and angular velocity $\omega$ of the track when the wheel is rolling. The track is deformed by the wheel pressure Fw and the sleeper pressure Fs1 and Fs2. At the same time, the wheel deforms due to the wheel-rail pressure Fr, and Figures 4(b) and 4(c) represent the undeformed and deformed states, respectively.

Regarding a point $(x, y)$ on the contact surface, if $z$ represents the pressure on the point, $u$ represents the displacement from the dashed line to the solid line along the normal direction, $q$ represents the distance of the dashed line when the point is not deformed, and $w$ represents its shape, the gap between the rear wheel and the track is $w=u+q$. Assume that $C$ is the contact surface and $E$ is the other external area, the geometric relationship shown in Figure 4 can be abbreviated as

$$
\begin{aligned}
& \forall(x, y) \in C, \quad w=0, \quad z \geq 0, \\
& \forall(x, y) \in E, \quad w>0, \quad z=0 .
\end{aligned}
$$

If the two-dimensional potential contact area with contact surface is discretized, users $m x \times m y$ grid is divided, and let $n$ represent the total number of grids; then

$$
u=T z, z, u \in R^{n}, T \in R^{n \times n}
$$

and the problem can be changed into a linear complementarity problem $\operatorname{LCP}(q, T)$; to find a pair $w, z \in R^{n}$, the following is satisfied

$$
w=T z+q \geq 0, z \geq 0, z^{T} w=0,
$$

where the coefficient matrix [32] $T$ is a Toeplitz matrix, satisfying 
TABle 2: Data of Example 9.

\begin{tabular}{lccc}
\hline Firm $i$ & $c_{i}$ & $L_{i}$ & $\beta_{i}$ \\
\hline 1 & 10 & 5 & 1.2 \\
2 & 8 & 5 & 1.1 \\
3 & 6 & 5 & 1 \\
4 & 4 & 5 & 0.9 \\
5 & 2 & 5 & 0.8 \\
\hline
\end{tabular}

TABle 3: Data of Example 10.

\begin{tabular}{lccc}
\hline Firm $i$ & $c_{i}$ & $L_{i}$ & $\beta_{i}$ \\
\hline 1 & 5 & 10 & 1.20 \\
2 & 3 & 10 & 1.00 \\
3 & 8 & 10 & 0.90 \\
4 & 5 & 10 & 0.60 \\
5 & 1 & 10 & 1.50 \\
6 & 3 & 10 & 1.00 \\
7 & 7 & 10 & 0.70 \\
8 & 4 & 10 & 1.10 \\
9 & 6 & 10 & 0.95 \\
10 & 3 & 10 & 0.75 \\
\hline
\end{tabular}

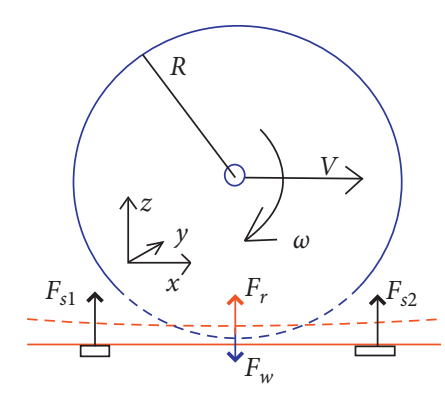

(a)

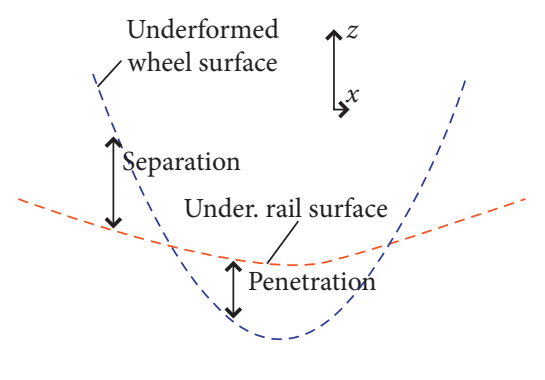

(b)

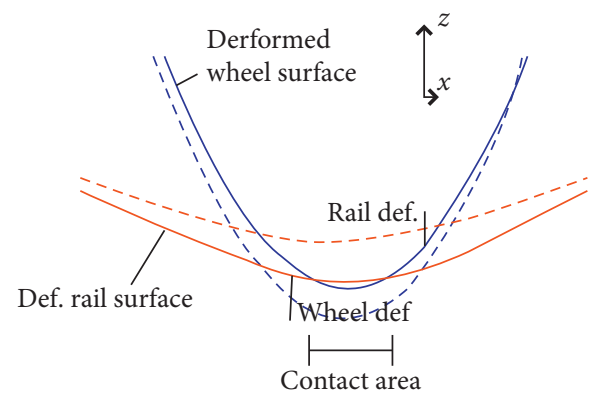

(c)

FIGURE 4: Schematic diagram of the two-dimensional contact problem [30].

$$
T=\left(\begin{array}{ccccc}
T_{0} & T_{-1} & \cdots & T_{2-n} & T_{1-n} \\
T_{1} & T_{0} & T_{-1} & \cdots & T_{2-n} \\
\vdots & T_{1} & T_{0} & \ddots & \vdots \\
T_{n-2} & \vdots & \ddots & \ddots & T_{-1} \\
T_{n-1} & T_{n-2} & \cdots & T_{1} & T_{0}
\end{array}\right) .
$$

Example 11. The diagonal element $T_{k}$ of the coefficient matrix $T$ is

$$
T_{k}= \begin{cases}2(1+k)^{-1.2}, & k \neq 0 \\ 2, & k=0 .\end{cases}
$$

Example 12. The diagonal element $T_{k}$ of the coefficient matrix $T$ is

$$
T_{k}=2^{-k}, \quad k=0,1, \ldots, n-1 .
$$

Example 13. The diagonal element $T_{k}$ of the coefficient matrix $T$ is

$$
T_{k}= \begin{cases}\left(\frac{19}{8}\right)+\left(\frac{1}{n}\right), & k=0 ; \\ -0.5, & k=1 ; \\ 0.25, & k=2 ; \\ \left(\frac{1}{16}\right), & k=3 ; \\ 0, & \text { else. }\end{cases}
$$

Table 4 shows the performance of Algorithm1 using different piecewise methods for practical application problems. From Figures 5 to 7 , it can be seen that Algorithm1 using 3-1 piecewise has a stronger ability to solve all the above problems than Algorithm 1 applying 4-1 piecewise. 
TABLE 4: Iterations, CPU time, and $x^{T} f(x)$ for problem 4.9-4.13.

\begin{tabular}{|c|c|c|c|c|c|c|c|}
\hline \multirow{2}{*}{ Problem } & \multirow{2}{*}{$\mathrm{x} 0$} & \multicolumn{3}{|c|}{ Algorithm 1 with $3-1$ piecewise } & \multicolumn{3}{|c|}{ Algorithm 1 with $4-1$ piecewise } \\
\hline & & Iter & CPU time & $\mathrm{XTF}(\mathrm{X})$ & Iter & CPU time & $\mathrm{XTF}(\mathrm{X})$ \\
\hline \multirow{2}{*}{4.9} & $20 *$ ones $(5,1)$ & 20 & 0.005197 & $-1.87 E-06$ & 23 & 0.00523 & $-1.49 E-06$ \\
\hline & $30 *$ ones $(5,1)$ & 18 & 0.010009 & $-2.28 E-06$ & 21 & 0.019381 & $-3.98 E-06$ \\
\hline \multirow{2}{*}{4.10} & $20 *$ ones $(5,1)$ & 45 & 0.033759 & $3.61 E-06$ & 43 & 0.050365 & $1.03 E-06$ \\
\hline & $30 *$ ones $(5,1)$ & 55 & 0.05047 & $7.62 E-07$ & 49 & 0.055852 & $-1.80 E-06$ \\
\hline \multirow{5}{*}{4.11} & ones $(100,1)$ & 13 & 0.052309 & $1.87 E-08$ & 15 & 0.089898 & $1.65 E-08$ \\
\hline & ones $(1024,1)$ & 14 & 2.080973 & $-3.66 E-09$ & 16 & 2.616348 & $1.94 E-09$ \\
\hline & ones $(4069,1)$ & 14 & 60.367315 & $1.05 E-09$ & 16 & 68.659795 & $8.54 E-09$ \\
\hline & ones $(8138,1)$ & 14 & 574.245627 & $3.05 E-09$ & 20 & 682.960276 & $-5.23 E-09$ \\
\hline & ones $(100,1)$ & 14 & 0.058579 & $1.96 E-09$ & 14 & 0.072436 & $-5.59 E-09$ \\
\hline \multirow{5}{*}{4.12} & ones $(1024,1)$ & 14 & 2.376266 & $-1.89 E-08$ & 14 & 2.348159 & $-4.86 E-09$ \\
\hline & ones $(4069,1)$ & 12 & 53.195688 & $-7.32 E-09$ & 14 & 61.321038 & $2.96 E-12$ \\
\hline & ones $(8138,1)$ & 17 & 519.846528 & $-2.89 E-08$ & 14 & 582.933657 & $3.25 E-11$ \\
\hline & ones $(100,1)$ & 17 & 0.060449 & $1.99 E-08$ & 19 & 0.0813907 & $4.00 E-09$ \\
\hline & ones $(1024,1)$ & 17 & 2.860266 & $5.62 E-08$ & 19 & 3.145843 & $8.61 E-09$ \\
\hline \multirow{2}{*}{4.13} & ones $(4069,1)$ & 17 & 74.532155 & $7.27 E-08$ & 19 & 82.960276 & $-2.45 E-09$ \\
\hline & ones $(8138,1)$ & 21 & 674.226819 & $2.02 E-08$ & 23 & 782.155632 & $5.12 E-09$ \\
\hline
\end{tabular}



FIgURE 5: Performance profile on CPU time for Algorithm1 with different piecewise functions.

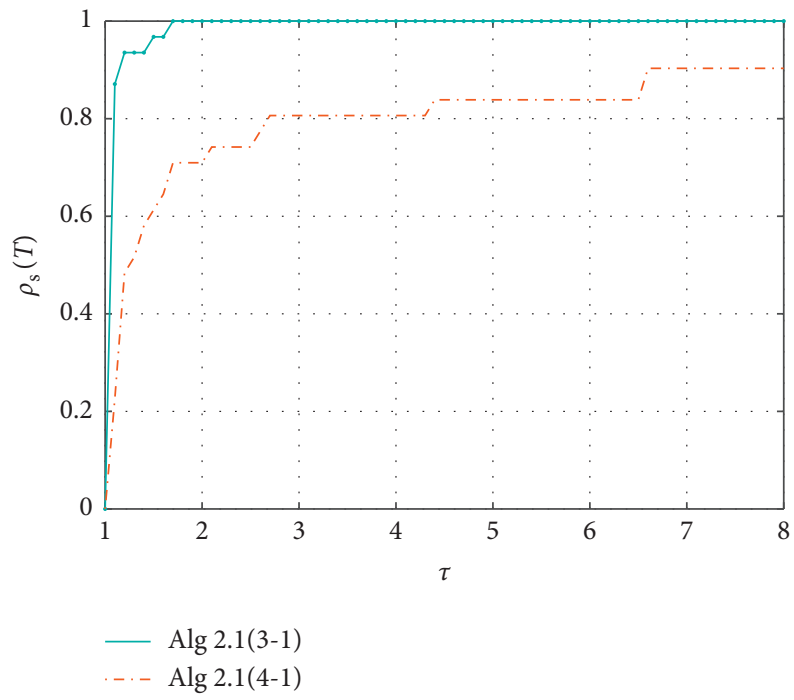

FIGURE 6: Performance profile on iterations for Algorithm1 with different piecewise functions. 


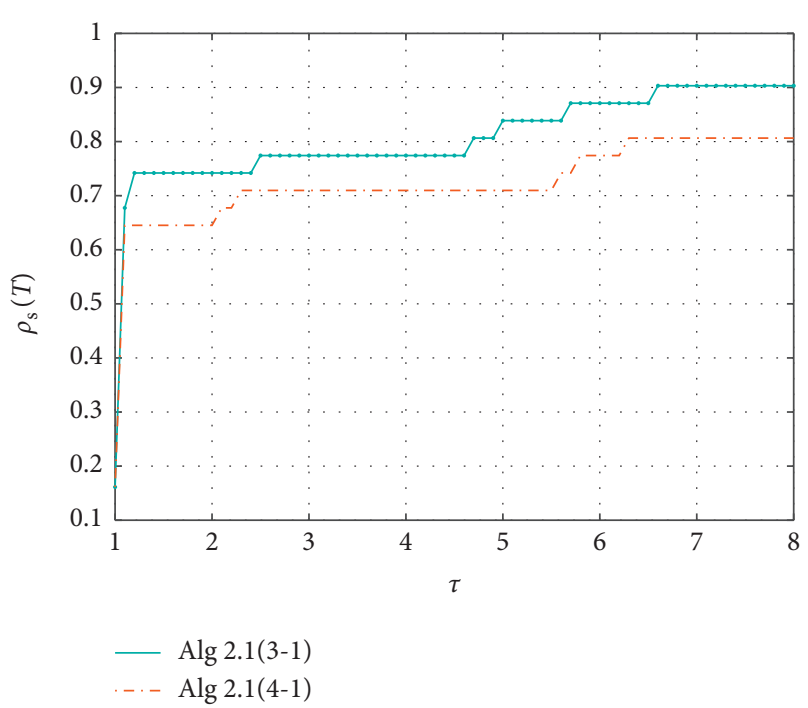

Figure 7: Performance profile on $x^{T} f(x)$ for Algorithm1 with different piecewise functions.

\section{Conclusion}

In this paper, by using 3-1 and 4-1 piecewise nonlinear complementarity problem functions, we reformulate the nonlinear complementarity problem into smooth equations. By using a new nonmonotone line search, a modified smooth Broyden-like algorithm is proposed and the global convergence of the proposed algorithm is obtained, and the numerical tests for some practical problems show the efficiency of the algorithm. How to get the local convergence under certain conditions is worth studying in the future.

\section{Data Availability}

The data used to support the findings of this study are included within the article.

\section{Conflicts of Interest}

The authors declare that there are no conflicts of interests regarding the publication of this paper.

\section{References}

[1] P. T. Harker and J.-S. Pang, "Finite-dimensional variational inequality and nonlinear complementarity problems: a survey of theory, algorithms and applications," Mathematical Programming, vol. 48, no. 1-3, pp. 161-220, 1990.

[2] J. J. Moré, "Global methods for nonlinear complementarity problems," Mathematics of Operations Research, vol. 21, no. 3, pp. 589-614, 1996.

[3] M. C. Ferris and J. S. Pang, "Engineering and economic applications of complementarity problems," Society of Indian Automobile Manufacturers Review, vol. 39, no. 4, pp. 669-713, 1997.

[4] A. Fischer, "A special Newton-type optimization method," Optimization, vol. 24, no. 3-4, pp. 269-284, 1992.
[5] B. Chen, X. Chen, and C. Kanzow, "A penalized FischerBurmeister NCP-function," Mathematical Programming, vol. 88, no. 1, pp. 211-216, 2000.

[6] D. Sun and L. Qi, "On NCP-functions," Computational Optimization and Applications, vol. 13, no. 1-3, pp. 201-220, 1999.

[7] T. Luca, F. Facchinei, and C. Kanzow, "A semismooth equation approach to the solution of nonlinear complementarity problems," Mathematical Programming, vol. 75, no. 3, pp. 407-439, 1996.

[8] H. Jiang and L. Qi, "A new nonsmooth equations approach to nonlinear complementarity problems," SIAM Journal on Control and Optimization, vol. 35, no. 1, Article ID 178193, 1997.

[9] T. Luca, F. Facchinei, and C. Kanzow, "A theoretical and numerical comparison of some semismooth algorithms for complementarity problems," Computational Optimization and Applications, vol. 16, pp. 173-205, 2000.

[10] L. Qi and Y. Yang, "NCP functions applied to Lagrangian globalization for the nonlinear complementarity problem," Journal of Global Optimization, vol. 24, Article ID 261283, 2002.

[11] J.-S. Pang and S. A. Gabriel, "NE/SQP: a robust algorithm for the nonlinear complementarity problem," Mathematical Programming, vol. 60, no. 1-3, pp. 295-337, 1993.

[12] C. Chen and O. L. Mangasarian, "A class of smoothing functions for nonlinear complementarity problems," Journal of Computational and Applied Mathematics, vol. 80, pp. 105-126, 1997.

[13] C. Kanzow and H. Pieper, "Jacobian smoothing methods for nonlinear complementarity problems," SIAM Journal on Optimization, vol. 9, no. 2, pp. 342-373, 1999.

[14] N. Krejic and S. Rapaji' c, "Globally convergent Jacobian smoothing inexact' Newton methods for NCP," Computational Optimization and Applications, vol. 41, pp. 243-261, 2008.

[15] L. Qi and D. H. Li, "A smoothing Newton method for nonlinear complementarity problems," Advanced Modeling and Optimization, vol. 13, no. 2, pp. 141-152, 2011.

[16] A. Liu, D. G. Pu, and D. Pu, "3-1 piecewise NCP function for new nonmonotone QP-free infeasible method," Journal of Robotics and Mechatronics, vol. 26, no. 5, pp. 566-572, 2014.

[17] S. Ke and D. Yang, "A smooth Newton method with 3-1 piecewise NCP function for generalized nonlinear complementarity problem," International Journal of Computer Mathematics, vol. 95, pp. 1703-1713, 2018.

[18] S. Ke and D. Yang, "A modified non-monotone method with 3-1 piecewise NCP function for nonlinear complementary problem," Computer Model. New Tech.vol. 21, no. 1, pp. 47-51, 2017.

[19] L. Qi and D. Sun, "Smoothing functions and smoothing Newton method for complementarity and variational inequality problems," Journal of Optimization Theory and Applications, vol. 113, no. 1, pp. 121-147, 2002.

[20] L. Qi, D. Sun, and G. Zhou, "A new look at Smoothing Newton method for complementarity problems and box constrained variational inequality problems," Antimicrobial resistance, vol. 97, p. 13, 1997.

[21] X. Chen, L. Qi, and D. Sun, "Global and superlinear convergence of the smoothing Newton method and its application to general box constrained variational inequalities," Mathematics of Computation, vol. 67, no. 222, pp. 519-541, 1998. 
[22] J. Tang, L. Dong, J. Zhou, and L. Fang, "A smoothing Newton method for nonlinear complementarity problems," Computational and Applied Mathematics, vol. 32, no. 1, pp. 107-118, 2013.

[23] C. Ma, X. Chen, and J. Tang, "On convergence of a smoothing Broyden-like method for -NCP," Nonlinear Analysis: Real World Applications, vol. 9, no. 3, pp. 899-911, 2008.

[24] B. Chen and C. Ma, "A new smoothing Broyden-like method for solving nonlinear complementarity problem with a $\mathrm{P} 0$ function," Journal of Global Optimization, vol. 51, no. 3, pp. 473-495, 2011.

[25] B. Fan, "A smoothing Broyden-like method with a nonmonotone derivative-free line search for nonlinear complementarity problems," Journal of Computational and Applied Mathematics, vol. 290, pp. 641-655, 2015.

[26] X. Y. Zheng, J. R. Shi, W. Yang, and Q. Y. Yin, "Nonmonotone smoothing Broyden-like method for generalized nonlinear complementarity problems," Journal of Applied Mathematics and Computing, vol. 26, pp. 566-572, 2014.

[27] J. S. Chen and S. H. Pan, "A regularization semismooth Newton method based on the generalized fischer-burmeister function for P0 -NCPs," Journal of Computational and Applied Mathematics, vol. 1, pp. 464-476, 2007.

[28] E. D. Dolan and J. J. Moré, "Benchmarking optimization software with performance profiles," Mathematical Programming, vol. 91, no. 2, pp. 201-213, 2002.

[29] L.-P. Zhang and Y. Zhou, "A note on economic equilibrium and financial networks," Acta Mathematicae Applicatae Sinica, English Series, vol. 30, no. 1, pp. 89-98, 2014.

[30] J. Zhao, E. A. H. Vollebregt, and C. W. Oosterlee, “A full multigrid method for linear complementarity problems arising from elastic normal contact problems," Mathematical Modelling and Analysis, vol. 19, no. 2, pp. 216-240, 2014.

[31] J. Zhao, E. A. H. Vollebregt, and C. W. Oosterlee, "A full multigrid method for linear complementarity problems arising from elastic normal contact problems," Mathematical Modelling and Analysis, vol. 19, no. 2, pp. 216-240, 2014.

[32] M. H. Wu and C. L. Li, "A preconditioned modulus-based matrix multisplitting block iteration method for the linear complementarity problems with Toeplitz matrix," Calcolo, vol. 56, p. 2, 2019. 